\title{
Intraoperative Electrocorticography in Temporal Lobe Epilepsy Surgery
}

\author{
David B. MacDonald and Neelan Pillay
}

\begin{abstract}
Although in clinical use for many years, the validity of intraoperative electrocorticography $(\mathrm{ECoG})$ in guiding resective temporal lobe epilepsy (TLE) surgery is uncertain. Advances in neuroimaging and extraoperative intracranial recordings have contributed greatly to the identification of epileptogenic lesions and cortex, clarifying the limitations of a brief intraoperative interictal recording. Studies of undifferentiated ECoG findings (which classify all interictal cortical spike discharges as equal) tend to not support this method. This article reviews ECoG and presents data from 86 TLE surgeries at the University of British Columbia suggesting that differentiation of ECoG features may enhance the contribution of this time honored method. Specifically, independent foci may be more important for epileptogenesis than synchronous foci, and postexcision activation appears to be a benign phenomenon, while residual spikes unaltered by the resection correlate with a greater proportion of seizure recurrence.
\end{abstract}

RÉSUMÉ: L'électrocorticographie peropératoire dans la chirurgie de l'épilepsie temporale. Bien que l'électrocorticographie (ÉCoG) peropératoire soit utilisée depuis plusieurs années, sa validité dans le guidage de la chirurgie de l'épilepsie temporale n'est pas établie. Les progrès de la neuroimagerie et les enregistrements intracrâniens extra-opératoires ont contribué beaucoup à l'identification de lésions épileptogènes et du cortex, clarifiant les limites d'un bref enregistrement interictal peropératoire. Des études d'observations ECoG indifférentiées (qui classifient toutes les décharges de pointes corticales interictales comme égales) tendent à ne pas supporter cette méthode. Cet article revoit l'ÉCoG et présente les données de 86 interventions chirurgicales pour épilepsie temporale, effectuées à l'Université de la Colombie Britannique, qui suggèrent que la différentiation des éléments de l'ÉCoG peuvent rehausser la contribution de cette méthode bien établie. Plus spécifiquement, les foyers indépendants peuvent être plus importants pour l'épileptogenèse que les foyers synchrones et l'activation postexcision semble être un phénomène bénin alors que les pointes résiduelles inchangées par la résection sont corrélées à une plus grande proportion de récidive des crises.

Can. J. Neurol. Sci. 2000; 27: Suppl. 1 - S85-S91

Initially, epilepsy surgery was lesion-based, but the development of electrocorticography $(\mathrm{ECoG})$ introduced an era of electrophysiologically-guided epilepsy surgery. Some investigators found cortical spike topography useful. They differentiated "primary" epileptiform abnormalities from those "conducted into relatively normal cortex from the primary epileptogenic area". ${ }^{1}$ They also distinguished "persistence of preexisting epileptogenic abnormality not included in the excision" from "the appearance ... of epileptiform abnormality in regions where, prior to the removal, this abnormality had been absent". ${ }^{1}$ Others found ECoG less useful.

Modern neuroimaging and extraoperative intracranial recording reemphasize lesions and direct attention to cortex involved in seizure onset. Consequently, the role and meaning of interictal ECoG spike topography in removal design remains controversial.
The purpose of this paper is mainly to introduce and compare and contrast the concepts of undifferentiated and differentiated ECoG in temporal lobe epilepsy surgery. These will be illustrated with previously unpublished data from the University of British Columbia (UBC) supporting ECoG differentiation. Since a comprehensive critical review of ECoG has recently been published, ${ }^{2}$ we have chosen to only briefly review the pathophysiology, methodology and current status of ECoG here.

From the Division of Clinical Neurophysiology, Vancouver Hospital \& Health Sciences Centre, Vancouver, BC, Canada (DBMcD) and Department of Clinical Neurosciences, University of Calgary, Calgary, AB, Canada (NP)

Reprint requests to: DB MacDonald, Division of Clinical Neurophysiology, Department of Neurosciences, King Faisal Specialist Hospital \& Research Center, MBC 76, PO Box 3354, Riyadh 11211, Saudi Arabia 


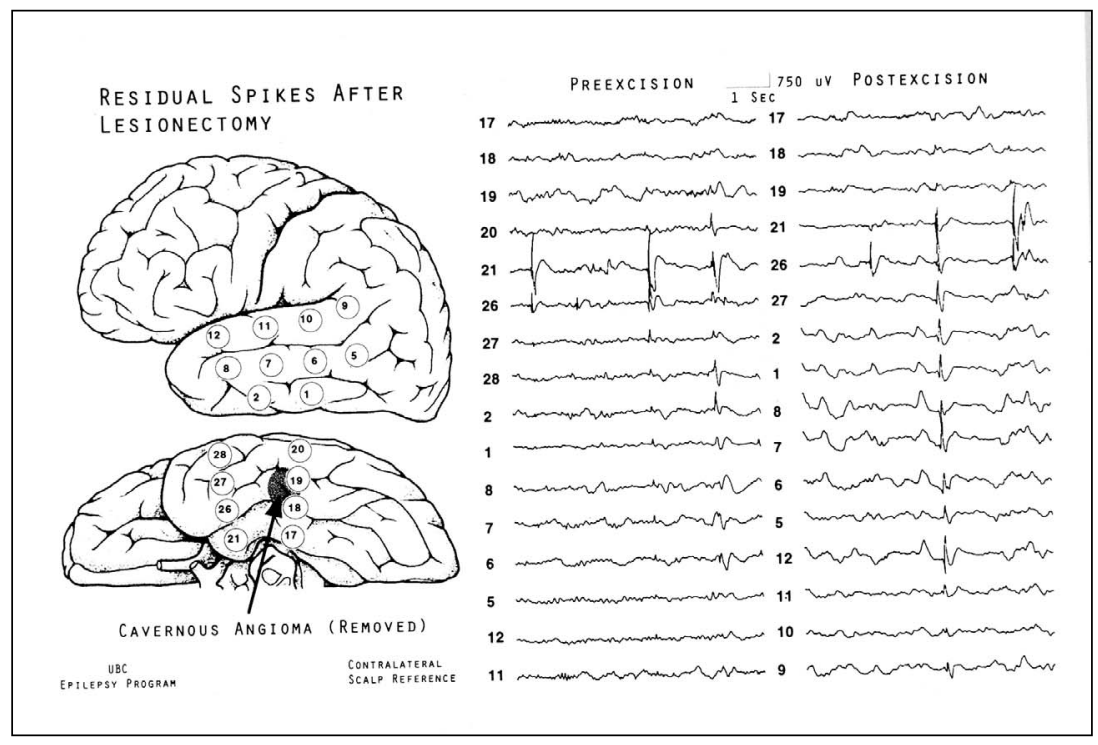

Figure 1: Complete removal (lesionectomy) of a dominant hemisphere cavernous angioma had no effect on independent foci at contacts 21 and 26 or widespread lateral synchronous foci. This is a clear example of residual spikes. The patient continued to have disabling complex partial seizures and focal left temporal spikes two years postoperatively.

\section{Pathophysiology and definitions}

Abnormal paroxysmal depolarizing shifts represent the interictal electropathophysiology of neurons in epileptic foci. ${ }^{3}$ Synchronous paroxysmal depolarizing shifts in groups of these neurons summate to produce EEG spikes which are uniquely relevant to the pathophysiology, diagnosis, and localization of partial seizure disorders.

An epileptogenic lesion is that structural abnormality which causes epileptic disruption of regional cerebral function. ${ }^{4}$ Excluding benign epilepsies of childhood, partial seizures imply a lesion, its identification a matter of technical limitation. Negative pathology in resected epilepsy surgery specimens or a residual lesion correlates with postoperative seizures. ${ }^{5}$

Coexistent with the epileptogenic lesion is the elusive epileptogenic zone, or cortex capable of generating seizures. ${ }^{4}$ It may be discrete or multifocal and independent. Proof of its complete removal manifests as the absence of postoperative seizures (including auras). When surgery is successful, it is not clear whether excision unnecessarily included additional cortex. Preoperative ictal intracranial ECoG estimates this zone, but the method has limitations, and is invasive.

The irritative zone is cortex containing interictal spikes, estimated by ECoG. ${ }^{4}$ It usually, but not always, contains the epileptogenic zone, and can be variable, multifocal, widespread, or bilateral and is often more extensive than the epileptogenic zone or lesion. The realization of this latter fact represents a significant limitation for ECoG in temporal lobe epilepsy surgery. It is incorrect to refer to the entire interictal irritative zone as the epileptogenic zone.

The irritative zone may be undifferentiated or differentiated. An undifferentiated map of this zone demarcates all cortical sites at which spikes are found. A differentiated map distinguishes spike discharges of different character, subdividing the irritative zone. There are no generally accepted criteria for such a differentiation. ${ }^{2}$ However, a simple descriptive method to do this intraoperatively, and used throughout this paper follows: Independent foci (IF) are spikes sometimes occurring only at a single or consistent group of electrodes. Due to spatial sampling limitations, it is possible that these spikes represent propagation from unrecorded cortex. Synchronous foci (SF) appear at other sites only in concert with identified independent foci. Residual spikes (RS) are unresected foci that continue to discharge with the same location, morphology and behavior during the postresection recording period (Figure 1). Post excision activation ${ }^{6}$ (PA) is the new appearance of epileptiform abnormality with a different location, morphology or behavior than before resection (Figure 2).

\section{ANESTHESIA}

Inhalational agents can suppress (halothane, high dose isoflurane and nitrous oxide) or activate (enflurane) spikes, and intravenous propofol may suppress spikes or induce burstsuppression and other patterns confounding interpretation. ${ }^{7}$ Local anesthesia essential for intraoperative functional mapping avoids these problems, but is arduous for the patient and creates greater technical difficulty. It may not be feasible for young children and some adults.

Although satisfactory ECoG is possible with light anesthesia (most commonly isoflurane and narcotic with or without nitrous oxide), its effect on ECoG is always in question. ${ }^{7}$ Discontinuing short acting anesthetics 10 - 20 minutes before recording with continued narcotic and neuromuscular blockade yields satisfactory ECoG but risks occasional awareness and recall. Most narcotics are considered to have little influence on spike activity, although there is some evidence for activation with alfentanil. ${ }^{8}$ 


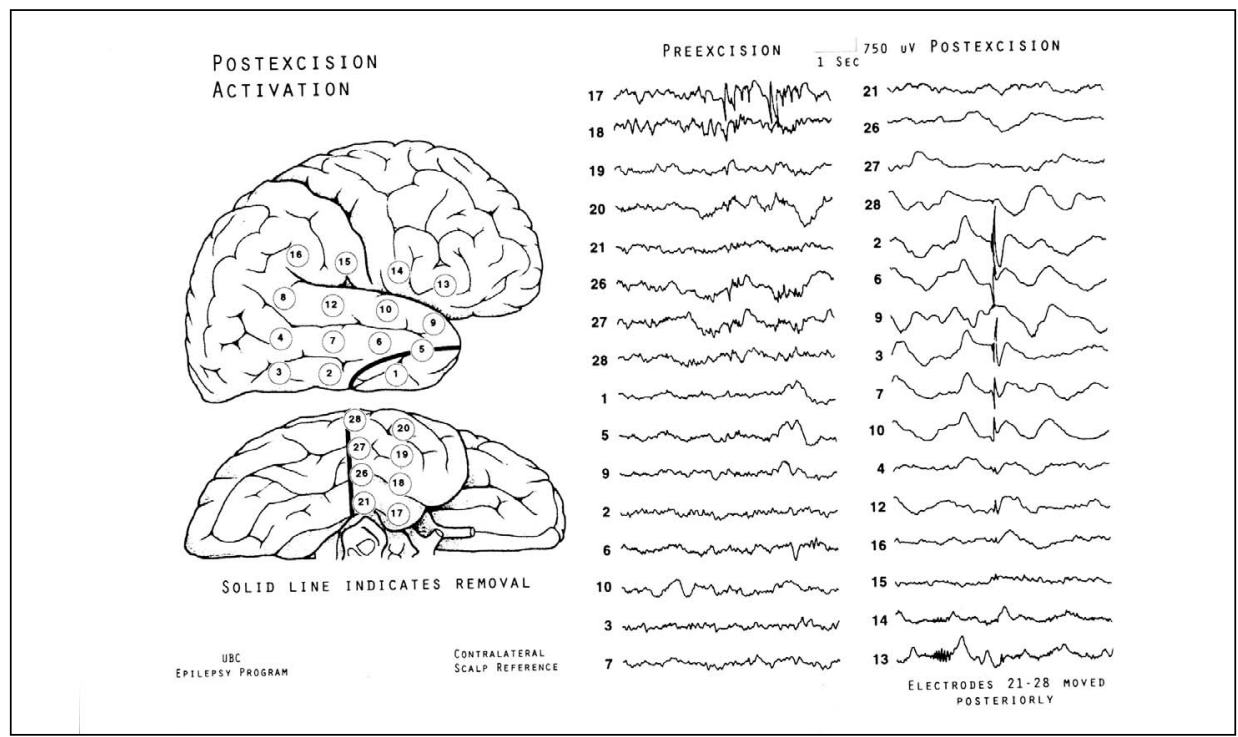

Figure 2: In this case of hippocampal sclerosis, the resection included the lesion and the entire congruent irritative zone, consisting of independent foci at contacts 17 and 18, and subtemporal synchronous foci not extending to the lateral surface. Removal of the inferior temporal tip provided access to mesial structures. The new lateral spike discharges in the postexcision recording are a typical example of postexcision activation. This patient was seizure and aura free off medication three years postoperatively. His EEG contained postoperative change without epileptiform abnormality.

Intravenous methohexital and other less commonly used activating agents (e.g. thiopentothal, etomidate and propofol) may increase cortical spike frequency and distribution. ${ }^{2,7}$ If used, it may be preferable to wait until after a thorough assessment of spontaneous ECoG, since there is no generally accepted approach to interpreting any expansion of the irritative zone. ${ }^{2}$

\section{TECHNICAL CONSIDERATIONS}

ECoG headsets consist of a rigid frame fixed at the craniotomy margin. These allow the surgeon to logically place individual electrodes according to the anatomy. Various reusable cortical contacts attach by springs to pivoting metal arms, and explore temporal and suprasylvian convexities. However, inferomesial cortex usually (but not always) contains the maximal epileptiform abnormalities ${ }^{9}$ (Figure 3).

Acute mesial depth electrodes sample amygdala and hippocampus, but require brain penetration, which some neurosurgeons prefer to avoid. Subtemporal silastic (silicone plastic) strip electrodes (preferably three or four) survey as far mesial as the parahippocampal gyrus and uncus, but cannot directly record the amygdala or hippocampus. Some investigators sample the hippocampal surface using a strip inserted in the opened temporal horn after partial resection. ${ }^{10}$

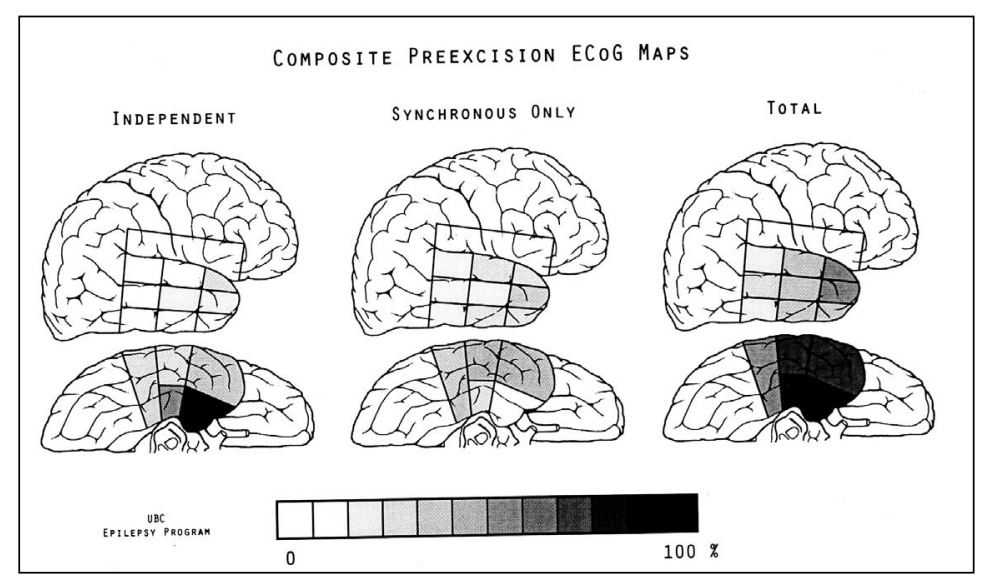

Figure 3: Composite maps of spontaneous preexcision ECoG features in 86 temporal lobe epilepsy surgeries at UBC. Shading represents the overall percentage of independent foci, synchronous foci and total foci found at each sector, when recorded. 
Whether or not postexcision activation influences such recordings is unknown. Some explore orbito-frontal cortex with a strip. ${ }^{9}$ Others employ strips or silastic grid electrode arrays on the lateral neocortex also, without a headset. ${ }^{11,12}$ This avoids a relatively cumbersome headset, and provides equal interelectrode spacing, but may be less adjustable to individual anatomy and the electrodes are not reusable.

Whatever method is chosen, anatomical geometric electrode arrays facilitate montage design and comparison. Recording adjacent and distant surfaces without spikes (including suprasylvian cortex) provides a better map of the irritative zone. Approximate reconstruction of the preexcision array after removal enhances postexcision interpretation.

ECoG records highly localized activity: spikes may be absent $1 \mathrm{~cm}$ away; negative and positive spike dipole ends can occur in adjacent electrodes. This differs from broad scalp EEG fields, and favors referential montages. A contralateral scalp reference is satisfactory. Orderly, anatomical channel grouping facilitates visual orientation. Instruments capable of simultaneously recording all available electrodes are desirable, otherwise adequate sampling requires montage adjustments. Demonstration of the independence or synchrony of convexity and inferomesial foci requires recording simultaneously at sufficient sites from each surface.

The recording bandpass should be 0.3 or $1 \mathrm{~Hz}$ to $70 \mathrm{~Hz}$ or higher, avoiding $60 \mathrm{~Hz}$ notch filters. Sensitivity should be $50-75$ $\mathrm{uV} / \mathrm{mm}$, otherwise clipping of spikes (often $1-3 \mathrm{mV}$ ) and excessive amplification of other activity impairs discrimination. Reasonable pre- and postexcision sampling requires up to 20 minutes each, depending on spike frequency.

\section{INTERPRETIVE PRINCIPLES}

The electroencephalographer designs and immediately interprets the recording considering all relevant information. The main task is to estimate the distribution and nature of the irritative zone active during the recording. Established criteria identify epileptiform abnormalities, with particular attention to distinction from background. Other abnormalities, such as focal polymorphic delta activity, signify dysfunction but do not map the irritative zone.

Commonly there are infero-mesial independent foci, with variable synchronous foci extending over other surfaces. This can suggest propagation, ${ }^{1}$ or volume conduction in cases of very low voltage synchronous potentials. In other cases, there are multifocal independent foci with or without additional synchronous foci.

It is desirable to distinguish residual spikes from postexcision activation. The later phenomenon includes spikes, sharp waves, periodic discharges, grouped lateral epileptiform discharges, burst-suppression patterns, and electrographic seizure discharges absent in the preexcison ECoG.

During cortical stimulation, the electroencephalographer immediately reports the occurrence of afterdischarges or induced seizure discharges. Cortical stimulation usually involves localized bipolar 50-60 Hz biphasic constant current stimulation of 2-10 milliamps for 1-5 seconds. ${ }^{2}$ Positive responses help identify functionally significant cortex. Afterdischarges may invalidate the findings. Occasionally, focal clinical seizures resembling the patient's spontaneous seizures may be induced. At times this may appear to be of localizing value, ${ }^{2}$ but seizure reproduction is not usually the main aim of ECoG.

\section{SIGNIFICANCE}

\section{Undifferentiated}

An undifferentiated map of the irritative zone delineates all recorded sites with any epileptiform abnormality considering all spikes equally. It may be reasonable to hope, but not conclude, that this contains the epileptogenic zone. When such an undifferentiated irritative zone is small and anatomically congruent to the lesion (if known) and hypothesized epileptogenic zone, its complete removal seems reasonable. However, removal of a large undifferentiated irritative zone extending well beyond the lesion or one not anatomically congruent with the lesion may be unfeasible or result in unnecessarily large resections. ${ }^{5,13}$ Several studies and a recent major review do not support such an undifferentiated approach to ECoG. ${ }^{2,11,12,14}$

A previously unpublished retrospective study of 86 temporal lobe epilepsy surgeries at UBC agrees with these conclusions (Figure 4). This study coded technically satisfactory ECoGs recorded between 1989 and 1995 and correlated the results to pathology and outcome according to Engel's classification. ${ }^{15}$ Great care was taken to distinguish (according to the above definitions) independent foci from synchronous foci in preexcision records, and residual spikes from postexcision activation in postexcision records.

Three categories of outcome were analyzed: A - IA (seizure and aura free), $\mathbf{B}$ - IA\&B (seizure-free with or without auras) and $\mathbf{C}-\mathrm{I}$ (free of disabling seizures with or without some seizure recurrence). All sequential surgeries performed for medically intractable temporal lobe epilepsy in the time period were evaluated. Anesthesia varied, but the great majority involved either light balanced anesthesia with isoflurane or discontinuing anesthetics (isoflurane or propofol) 10-20 minutes before

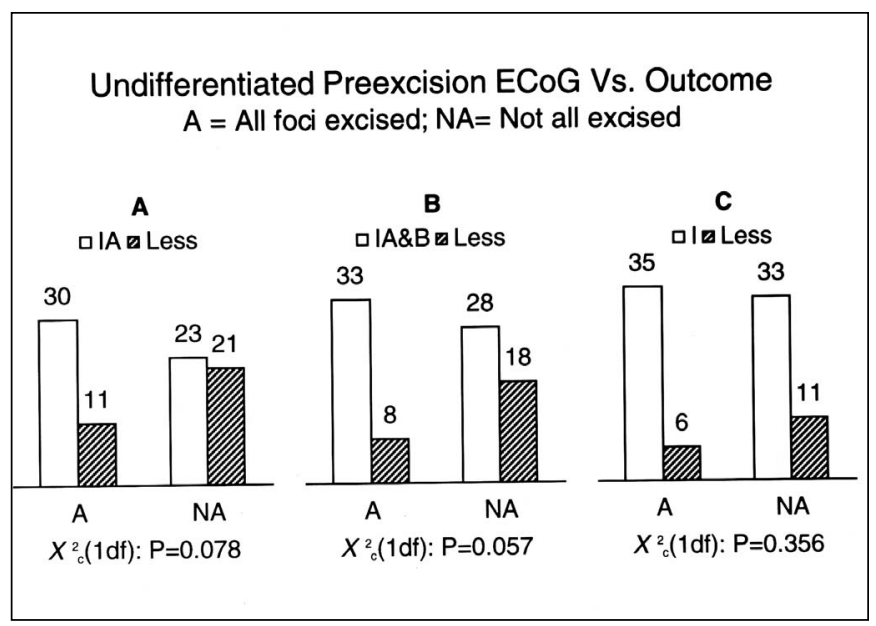

Figure 4: In a UBC series, the completeness of undifferentiated irritative zone removal did not statistically correlate to seizure outcome proportions, particularly for disabling seizures $(C)$. 


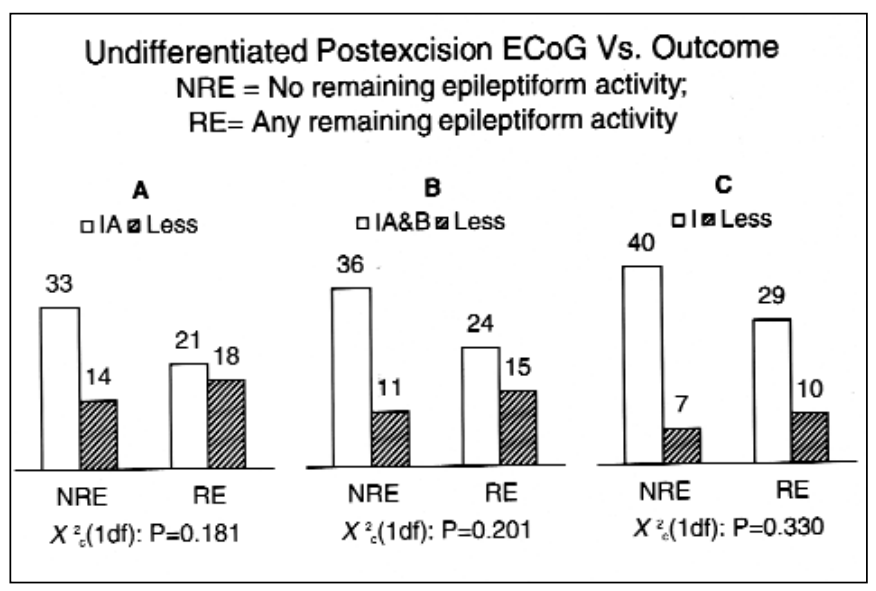

Figure 5: The presence of any undifferentiated remaining epileptiform activity did not statistically correlate to seizure outcome proportions (A, $B$, and $C$ ).

recording, with continued narcotic and neuromuscular blockade. Exclusion criteria included: negative results (one case, during low dose propofol infusion), postexcision recording time less than five minutes (range, 5-29, mean 13.3 minutes) and technically unsatisfactory recordings (e.g., exclusively bipolar recording, excessive recording gain, serious unresolved technical problems). All recordings used a standardized array of temporal and suprasylvian convexity contacts using a headset, and two to four subtemporal strips. Twelve included a strip electrode on the hippocampus after partial resection to open the temporal horn. These were too few to analyze separately, but ten revealed independent spike activity. A single board certified electroencephalographer (DBM) who was blind to the outcome of the majority, but not all patients, reviewed all records. Pathology and outcome data were collected after all ECoGs were coded. Statistical analysis utilized the Chi-square statistic for difference in proportions in $2 \times 2$ (with Yate's correction) or $2 \times 3$ contingency tables. Follow up was 1.8 - 8.7 (mean 4.6) years.

This study forms the basis for Figures $3-9$. The extent of resection of the undifferentiated irritative zone did not correlate to seizure outcome.

If postexcision spikes predict a greater chance of seizure recurrence, then additional removals guided by ECoG could be reasonable. However, the presence of any undifferentiated (considering residual spikes and postexcision activation equally) epileptiform activity in the postexcision ECoG fails to correlate to seizure outcome. ${ }^{2,11,12,14}$ Data from UBC agree with these conclusions (Figure 5).

\section{Differentiated}

Differentiating independent foci from synchronous foci and residual spikes from postexcision activation in ECoG tends to find more meaningful correlations. ${ }^{1}$ This agrees with intuition and reports of the predictive power of postoperative EEG. ${ }^{16,17}$ Data from UBC support the ideas that independent foci may be more significant for epileptogenesis than synchronous foci, and that postexcision activation may be benign, while residual spikes correlate with a greater chance of seizure recurrence (Figures 6 and 7). In this study, unresected independent foci often persisted

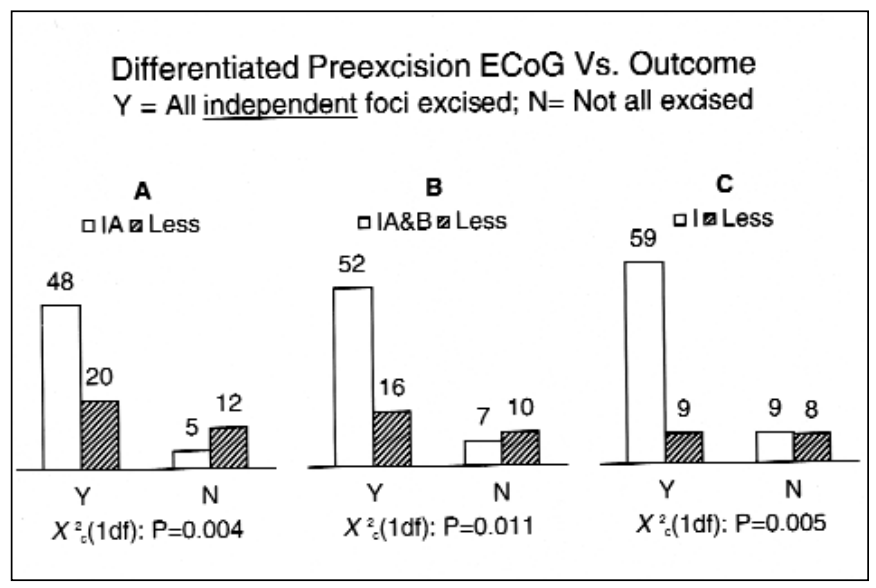

Figure 6: When some independent foci were unresected, a greater proportion of patients experienced some degree of seizure recurrence, including disabling postoperative seizures $(A, B$, and $C)$.

as residual spikes, while unresected synchronous foci often disappeared after excision of independent foci. The extent of resection of synchronous foci after complete removal of independent foci did not correlate with outcome. It may be advisable to include independent foci in the resection when possible and clinically indicated. The additional inclusion of extensive synchronous foci is probably unnecessary.

Novel postexcision epileptiform features may be acute reactions to the resection and do not correlate well with seizure recurrence. ${ }^{1,11,18-21}$ Similar ECoG findings can follow frontal leukotomy, ${ }^{19}$ and it is well known that transient epileptiform EEG abnormalities can arise in acute or subacute destructive cerebral lesions, without necessarily producing clinical seizures. It would be interesting to know if this phenomenon occurs in nonepileptic temporal lobe surgeries. "Chasing" postexcision activation with additional resections is a questionable practice.

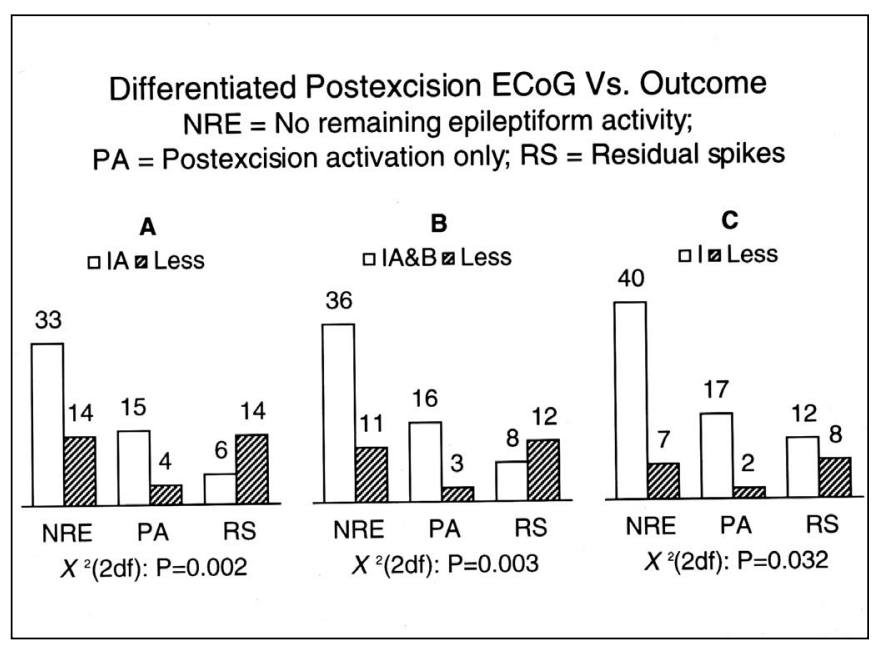

Figure 7: Residual spikes statistically correlated to a greater proportion of seizure recurrence, including disabling seizures $(A, B$, and $C)$. Postexcision activation appears benign in all Engel outcome categories. 


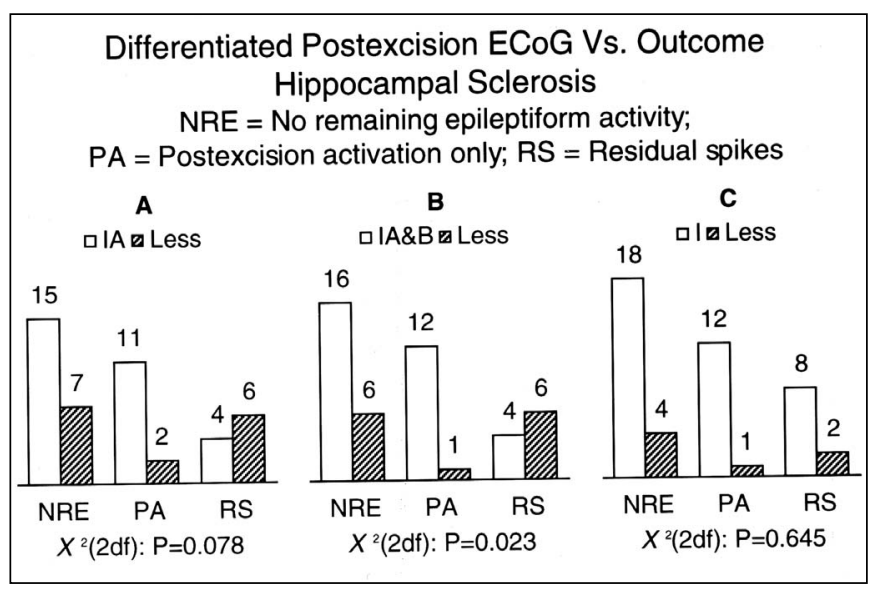

Figure 8: While some degree of nondisabling postoperative seizures statistically correlated to residual spikes $(B)$, there was no greater proportion of patients experiencing disabling postoperative seizures when residual spikes were present after resection of hippocampal sclerosis. (C).

\section{Hippocampal sclerosis}

Hippocampal sclerosis itself may be all or most of the epileptogenic zone. ECoG features, while usually congruent, may also include distant epileptiform abnormality. ECoG results may be less meaningful (though not necessarily meaningless) regarding outcome after removing the lesion (possibly the epileptogenic zone). Data from UBC tend to support this idea (Figure 8). This may account for the success of small inferomesial resections or amygdalohippocampectomy in some of these patients regardless of ECoG findings. Whether it is valuable to tailor the resection of additional cortex according to ECoG remains uncertain. These considerations may also apply to some other electrically active lesions such as focal cortical dysplasias.

\section{Other lesions}

Certain lesions may generate seizures through disturbance of adjacent or possibly even distant cortex. Lesionectomy alone may be successful, but carries a significant failure rate. ${ }^{22}$ Some data support a role for ECoG in these patients. ${ }^{22,23,24}$ Data from UBC agree with this idea (Figure 9). Although ECoG may be congruent to the vicinity of the lesion, there are often, but not always, inferomesial independent foci even if the lesion is not mesial. Removal of histologically intact functional mesial structures may produce mild modality-specific memory disturbances, ${ }^{22}$ most important in the dominant hemisphere. The surgeon must sometimes decide whether to risk this for the greatest chance of seizure-freedom or hope for successful lesionectomy. If independent foci are unresected, ECoG may provide some basis for understanding any seizure recurrence, and a rationale for a second surgery. The absence of inferomesial independent foci in these patients may provide some basis for avoiding additional mesial resection. . $2,25^{2}$

\section{ROLE}

Surgical philosophy and preoperative evaluation may predetermine the resection, including amygdalohippocampec-

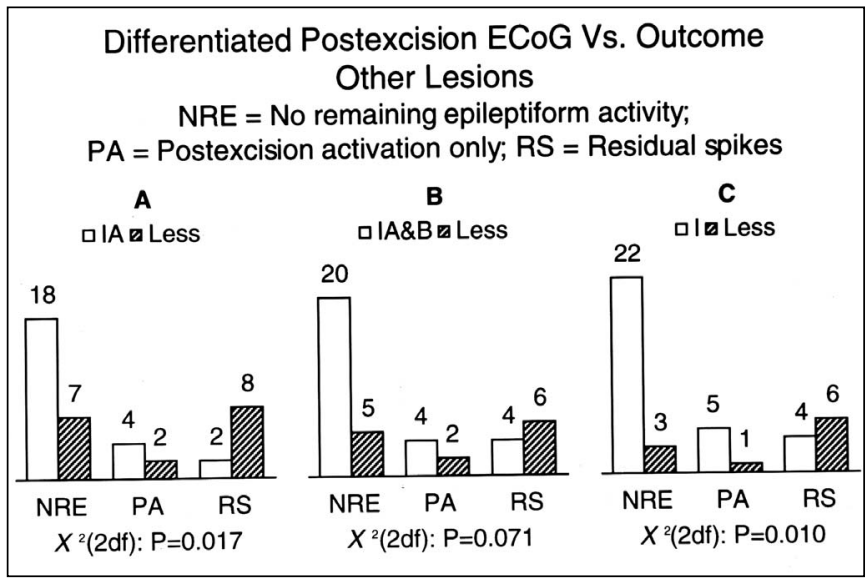

Figure 9: A greater proportion of patients with other lesions experienced some degree of postoperative seizures ( $A$ and $C)$, including disabling seizures $(C)$, if residual spikes were present.

tomy, lesionectomy, en-bloc resections (lobectomy), and removals based on extraoperative ECoG. In these circumstances, intraoperative ECoG is academic but to advance understanding and to enhance analysis of failures, it remains reasonable, if unnecessary, to record these studies.

When imaging fails to disclose a lesion (including hippocampal sclerosis), and without extraoperative ictal ECoG recordings, intraoperative ECoG becomes more important in the attempt to localize epileptogenic cortex.

If the surgeon decides to incorporate $\mathrm{ECoG}$, it is important to view it as only one element in removal design. One must realize the limitations of this and other methods and consider all available information. Until routinely definitive preoperative identification of the epileptogenic zone is available, intraoperative ECoG will continue to be relevant for these patients.

Afterdischarges following cortical stimulation invalidate clinical responses, and induced habitual clinical seizures may have localizing value. Therefore, ECoG remains essential for functional mapping.

\section{CONCLUSIONS}

Intraoperative ECoG offers the advantage of providing unique and relevant pathophysiologic information of some localizing value. However, the irritative zone is often more extensive than the epileptogenic zone or lesion, and the removal itself may incite postexcision epileptiform abnormalities. Consequently, an undifferentiated approach to ECoG fails to correlate well with seizure outcome, and casts doubt on the validity of this method. In the UBC series presented here, differentiating independent foci from synchronous foci in the irritative zone, and residual spikes from postexcision activation in postexcision recordings, appears to provide more meaningful correlations to outcome. Even so, the relationship of these features with outcome in individual cases remains imperfect. There may be more powerful methods of differentiating ECoG features that remain to be identified. Future studies should 
address methods of ECoG differentiation and the relationship between the nature of the epileptogenic lesion, ECoG results, and outcome. ${ }^{26}$ Digital recording with computerized analysis might allow intraoperative quantification of ECoG including measurements of spike frequency, timing, cumulative voltage, or other features of possible significance. ${ }^{2}$

While there are some theoretical risks in the prolongation of temporal lobe epilepsy surgery in order to obtain an ECoG, there are no reports of complications, ${ }^{2}$ and therefore, the moderate benefits continue to seem justifiable.

\section{REFERENCES}

1. Gloor P. Contributions of electroencephalography and electrocorticography to the neurosurgical treatment of the epilepsies. In: Purpura DP, Penry JK, Walter RD, eds. Advances in Neurology, Vol. 8, New York: Raven Press, 1975: 59-105.

2. Chatrian GE, Quesney LF, Intraoperative Electrocorticography. In: Engel J, Pedley TA, eds. Epilepsy - A Comprehensive Textbook, Vol. 2, Philadelphia - New York: Lippincott - Raven, 1998:17491765.

3. Speckmann EJ, Elger CE. Introduction to the neurophysiological basis of the EEG and DC potentials. In: Niedermeyer E, Lopes da Silva F, eds. Electroencephalography. Baltimore-Munich: Urban \& Schwarzenberg, 1987: 1-13.

4. Luders HO, Awad I, Conceptual Considerations. In: Luders HO, ed. Epilepsy Surgery. New York: Raven Press, 1992: 51-62.

5. Clarke DB, Olivier A, Andermann F, Fish D. Surgical treatment of epilepsy: the problem of lesion/focus incongruence. Surg Neurol 1996; 46: 579-586.

6. Jasper HH, Electrocorticography. In: Penfield W, Jasper HH, eds. Epilepsy and the functional anatomy of the human brain. Boston: Little, Brown, 1954; 692-738.

7. Kraemer DL, Spencer DD. Anesthesia in epilepsy surgery. In: Engel J, ed. Surgical Treatment of the Epilepsies. 2nd ed., New York: Raven Press, 1993: 527-538.

8. Cascino GD, So EL, Sharbrough FW, et al. Alfentanil-induced epileptiform activity in patients with partial epilepsy. J Clin Neurophysiol 1993; 10:520-525.

9. Tsai ML, Chatrian GE, et al. Electrocorticography in patients with medically intractable temporal lobe seizures I. Quantification of epileptiform discharges prior to resective surgery. Electroencephalogr Clin Neurophysiol 1993; 87(1): 10-24.

10. Tsai ML, Chatrian GE, et al. Electrocorticography in patients with medically intractable temporal lobe seizures II. Quantification of epileptiform discharges following successive stages of resective surgery. Electroencephalogr Clin Neurophysiol 1993; 87(1): 2537.

11. Tran TA, Spencer SS, et al. Significance of spikes recorded on electrocorticography in nonlesional medial temporal lobe epilepsy. Ann Neurol 1995; 38(5): 763-770.

12. Cascino GD, Trenerry MR, et al. Electrocorticography and temporal lobe epilepsy: relationship to quantitative MRI and operative outcome. Epilepsia 1995; 36(7): 692-696.

13. Freid I. Management of low-grade gliomas: results of resections without electrocorticography. Clin Neurosurg 1995; 42: 456-463.

14. Kanazawa O, Blume WT, Girvin JP. Significance of spikes at temporal lobe electrocorticography. Epilepsia 1996; 37(1): 50-55.

15. Engel J, Van Ness PC, et al. Outcome with respect to epileptic seizures. In: Engel J, ed. Surgical Treatment of the Epilepsies. 2nd ed., New York: Raven Press, 1993: 609-621.

16. Tuunainen A, Nousiainen U, et al. Postoperative EEG and electrocorticography: relation to clinical outcome in patients with temporal lobe surgery. Epilepsia 1994; 35(6): 1165-1173.

17. Patrick S, Berg A, Spencer SS. EEG and seizure outcome after epilepsy surgery. Epilepsia 1995; 36(3): 236-240.

18. Cendes F, Dubeau F, et al. Increased neocortical spiking and surgical outcome after selective amygdalohippocampectomy. Epilepsy Res 1993; 16(3): 195-206.

19. Hosain S, Burton L, Fraser R, Labar D. Focal suppression-burst on electrocorticography after temporal lobectomy. Neurology 1995; 45(12): 2276-2278.

20. Wennberg RA, Quesney LF, et al., Induction of burst-suppression and activation of epileptiform activity after methohexital and selective amygdalo-hippocampectomy. Electroencephalogr Clin Neurophysiol 1997; 102(5): 443-451.

21. Wennberg RA, Quesney LF, et al., Increased neocortical spiking and surgical outcome after selective amygdalohippocampectomy. Electroencephalogr Clin Neurophysiol Suppl 1998; 48: 105-111.

22. Jooma R, Yeh HS, et al. Lesionectomy versus electrophysiologically guided resection for temporal lobe tumors manifesting with complex partial seizures. J Neurosurg 1995; 83(2): 231-236.

23. Pilcher WH, Silgergeld DL, et al. Intraoperative electrocorticography during tumor resection: impact on seizure outcome in patients with gangliogliomas. J Neurosurg 1993; 78(6): 891-902.

24. Berger MS, Ghatan S, et al. Low-grade gliomas associated with intractable epilepsy: seizure outcome utilizing electrocorticography during tumor resection. J Neurosurg 1993; 79(1): 6269.

25. Kanner AM, et al. Tailored anterior temporal lobectomy. Relation between extent of resection of mesial structures and postsurgical outcome. Arch Neurol 1995 Feb; 52(2): 173-178.

26. Binnie CD, McBride MC, et al. Electrocorticography and stimulation. Acta Neurol Scand Suppl 1994; 152: 74-82. 